\title{
Assamese Digit Recognition with Feed Forward Neural Network
}

\author{
Kalyanbrat Medhi \\ Department of Computer Science \\ Gauhati University \\ Guwahati, India
}

\begin{abstract}
The aim of this paper is to design a recognizer to recognize Assamese digits using feed forward neural network. The recognizer crops the individual digits of the image using bounding box method and extracts the feature. In the present study zoning is used to obtain necessary feature vector. This feature is provided as input to the classifier and the network is trained with backpropagation training algorithm with two hidden layer. The recognition rate of printed digits is $98 \%$, including multi size, bold and italics fonts. In case of handwritten digits recognition rate is $70.6 \%$.
\end{abstract}

\section{General Terms}

Pattern Recognition

\section{Keywords}

Assamese digits Recognition, Feed Forward Neural Network, Zoning, Back Propagation.

\section{INTRODUCTION}

Now a day's one of the most important applications of pattern recognition is to design character recognition system that capable of recognizing human or machine written characters. These systems can produce editable document and thus reduce the human effort. Since the advancement the digitalization age, character recognition by machine remains as an active research field. Although digits are subset of any languages' character sets they are very important to many applications. Many applications require only digit identification. Automatic printed digit recognition has a variety of applications as interface between man and machine. Areas of application are numerous ranging from OCR (Optical Character Recognition), license plate recognition of vehicles, price recognition of items in shopping mall, bank's check number reading, postal number reading etc. In general, there are two categories of character recognition systems; recognize while writing it, called on-line recognition technique or recognize after writing it, called off-line recognition technique. In this paper we discuss about an experiment which uses the latter approach i.e. off-line technique. There are various off-line character recognition systems available which may be specific or generic. The generic systems are capable of processing any type of scanned document, which may contain any font or even graphics also and produce equivalent editable text. Due to its lots of functionality the performance of such type of systems is not always high. The specific recognition systems are used for special tasks only, such as specific font is to be recognized and due to limited font processing accuracy is higher than the generic system when use for specific purpose.

Most commercial applications of OCR are available for English, Chinese, Roman, Japanese and Arabic language. These OCR are generic type capable of recognizing characters with different fonts and sizes even intermixing text and graphics. The OCR systems have been already developed for

\author{
Sanjib Kr. Kalita, Ph.D. \\ Assistant Prof, Dept. of Computer Science \\ Gauhati University \\ Guwahati, India
}

[12] Bangla, [22] Devanagari, [23] Gurumukhi, [24] Kannada, [25] Malayalam, [26] Tamil, [27] Telugu [1].

\section{MOTIVATION}

Literature review reveals that studies on Bangla and Telugu are carried out by several researchers [12, 29-32, 33-35]. But less efforts has been put to develop character recognition tool for the North-Eastern languages, particularly the Assamese language. The lack of effective research work in Assamese language motivated us to develop a digit recognizer.

\section{THEORETICAL BACKGROUND}

A document is scanned by an optical scanner to produces an image which is not in editable format. In general, character recognition system goes through the following steps as shown in figure 1.

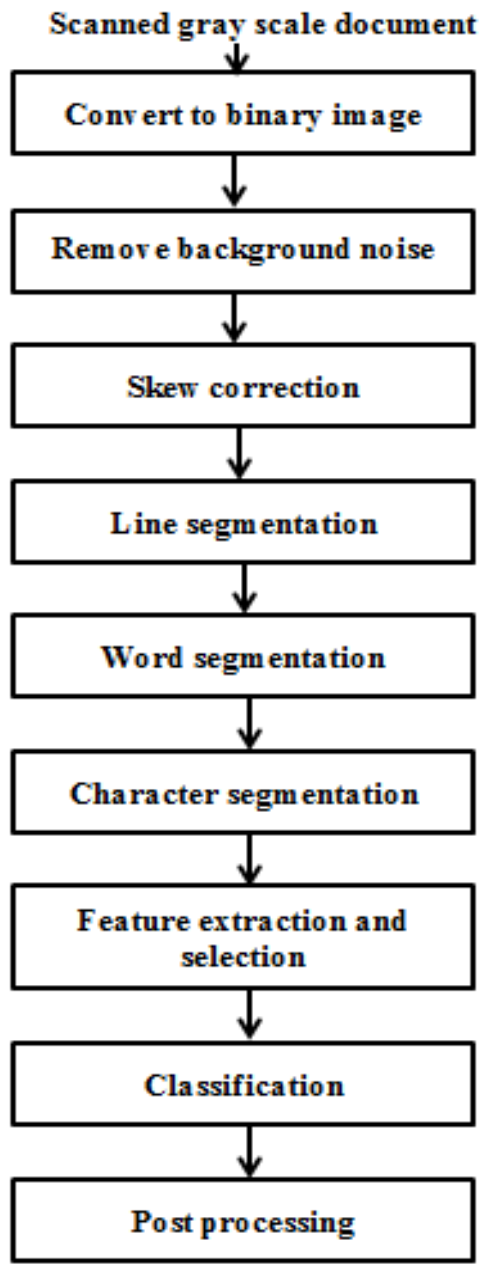

Figure 1: Block diagram of character recognition system 
The scanned document image is converted to binary image followed by noise remove and skew correction. Then segmentation of line, word and character is performed. In the next step, certain features are extracted so that for each character the feature sets are different. In classification stage, the character having certain features is put in particular class. Post processing is an optional process, but important for unconstrained handwriting recognition and if the classifier put a character to more than one class.

\subsection{Image Acquisition}

The very first task of $\mathrm{CR}$ system is to obtain the image patterns. Image may be obtained by scanning document as well as from various standard datasets. In the earlier process, the paper needs to be digitized by scanning it with scanner in good resolution (300dpi or more) and save as any image format. In present study, images are acquired by scanning document in 300dpi. The image obtained through both processes needs to go through lots of preprocessing steps. The basic goal of preprocessing is to produce neat and clean version input image for efficient feature extraction. The following figure shows a portion of scanned image.

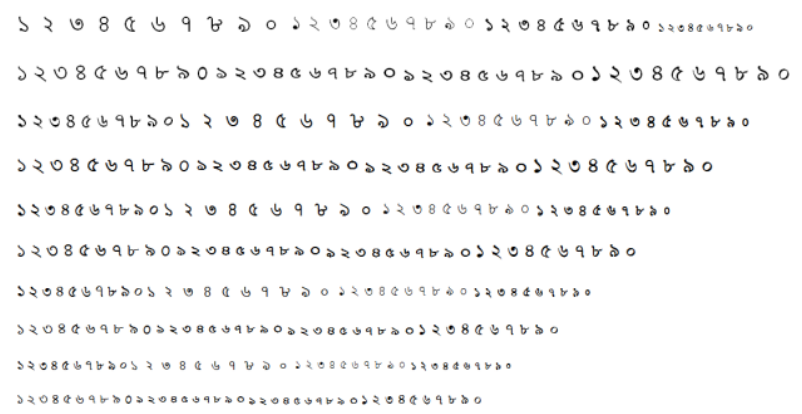

Figure 2: A scanned document image

\subsection{Binarization}

It is used to extract text from low quality image background such as poor contrast, non-uniform background, and random noise due to limited sensitivity of sensor. The gray scale images are converted to binary images using appropriate threshold that may be fixed or dynamically calculated from the image. All the pixels which threshold value is greater than the given threshold value are set as 1 otherwise as 0 . In this experiment 0.9 threshold value is used. The following figure shows image before binarization and after binarization.

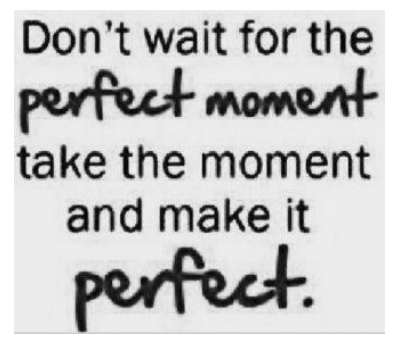

a

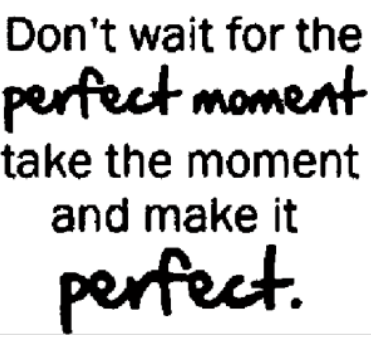

b
Figure 3: An original image a (left) and image b after binarization (right).

\subsection{Noise Removing}

Digital images are affected by several types of noise; which occurs during acquisition process due to printer, print quality, scanner's or camera's sensor heat, age of the document, etc. It is better to filter this noise before the OCR attempt to recognize the image, as noise reduces the performance of

reorganization system. Noise can be removed by using linear filtering, medial filtering and adaptive filtering [21]. Morphological openings and closings are also useful for the smoothing of gray-scale images [13]. This technique is used in this recognizer. Here is example of noise removal from binary image by morphologically opening the image in the following figure 3 .

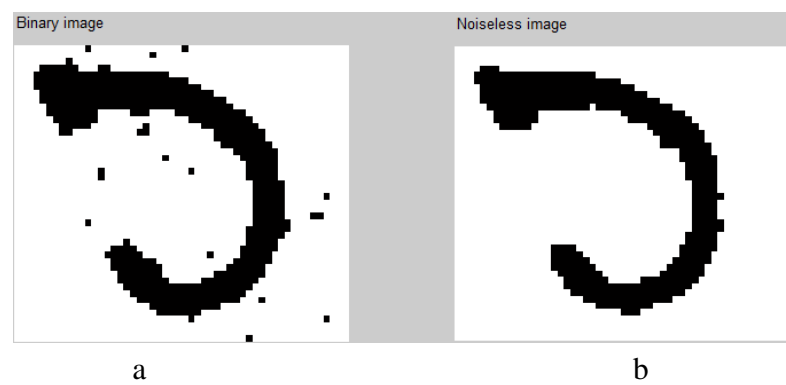

Figure 4: An original binary image a (left) with noise, and image $b$ after noise removal (right).

\subsection{Skew Detection and Correction}

When a document is fed in to the scanner either by man or machine, a few degrees of tilt (skew) is unavoidable. Skew angle is the angle between the lines of text in the digital image and the horizontal direction. Figure 5 shows an image with skew.

\section{y-critical system istinction can be tes. The mission haviour while the y controller when more, the aims of nission controller ed - this will also zr into an unsafe led with avoiding unsafe states that}

$y$-critical system istinction can be tes. The mission haviour while the y controller when more, the aims of nission controller ed - this will also er into an unsafe led with avoiding unsafe states that

\section{$\mathrm{b}$}

Figure 5: A skew less image a (left), and image with skew in image $b$ (right)

There exist several techniques for skew estimation. One technique of skew estimation considers the projection profile of the document; another class of approach is based on nearest neighbor clustering of connected components. Literatures exist for skew detection and correction in [14-17].

\subsection{Line, Word and Character Segmentation}

The segmentation process plays a crucial role in the overall process of recognition of printed and handwritten characters. According to the remark of Marosi [18] a reliable character segmentation method is more important than the recognition performance of the classifier.

\subsection{Thinning}

Thinning is a morphological operation in which a one-pixelwidth representation of an object is obtained. It successively removes the boundaries of foreground objects as defined, 
without affecting the connectedness of pixels or the ends of lines. Thinning is normally applied to only binary images to produce skeleton of the binary image. The following figure 6(a) shows an image and its thin format in figure 6(b).

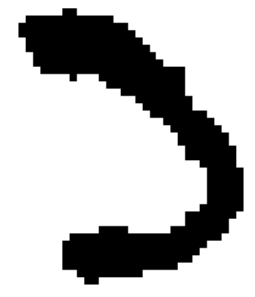

a

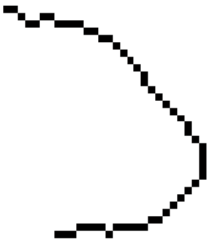

b
Figure 6: An image (left) before thinning, and (right) after thinning.

\subsection{Feature Extraction and Selection}

Feature extraction is process of simplifying the amount of resources that requires for describing a large set of data accurately. It is another crucial step to design OCR systems with good performance. Some of techniques used for feature extraction are projection histograms, moments, zoning, ntuples, crossings and distances etc. In present study zoning is used as feature for reorganization of digits.

\subsection{Classification}

Classification is a process to categories similar object. It is one of the most important components of OCR system. A number of classification methods are used; such as neural networks classifier, tree based classifier, Hidden Markova Model (HMM) based classifier etc. Artificial neural networks can handle non-convex decisions. Multi-layer feed forward neural network that trained with a back-propagation learning algorithm is the most popular neural network [19]. The Radial-Basis Function (RBF) network can give competitive accuracy with the Multi-layer perceptrons (MLP) when training all parameters by error minimization [20]. Limitation of perceptrons is that it can classify only linearly separable sets of vectors in finite time; otherwise all vectors will not be classified properly. Input vectors can be said linearly separable if a plane or a straight line can separate them into their correct categories. The feed forward neural network is able to overcome this situation.

\subsection{Post-Processing}

It improves the accuracy of system. The outputs of the character recognition classifier unit may have several character candidates. The post processing helps to determine the best combination of characters out of candidate characters.

\subsection{Feed forward Neural Network Classifier}

Feed forward neural network is a special type of ANN which can be used for classification of pattern image. It consists of a number of layers 1, $2 \ldots \mathrm{L}$, where each layer contains many neurons (or nodes). Every neuron in layer 1 receives input from all the neurons of layer 1-1and propagates the output of every neuron of layer 1 to layer $1+1$. The layer 1 is called input layer which receives the feature vector of pattern image and the layer $\mathrm{L}$ is called output layer which gives the result of classification. All the layers between the two layers are called hidden layers. The input layer consist of as many neurons as the length of input column vector, while number of neurons in output layer is equals to number of pattern class. In this network there is no feedback link from higher layer to lower layers and this is why it is called feed forward neural networks. Each of the links may have a different weight, which indicates the knowledge of a network. Data fed into the input layer as a column vector propagate through the entire hidden layers until it arrives at the outputs layer. An input to the network may be either a raw image or some specific features that are extracted from the pattern image. Features extraction is a crucial phase and it is application dependent. Usually the weights on links are assigned in random way in the beginning $[4,5]$. Here is a diagram of simple feed forward neural network with one hidden layer in figure 7.

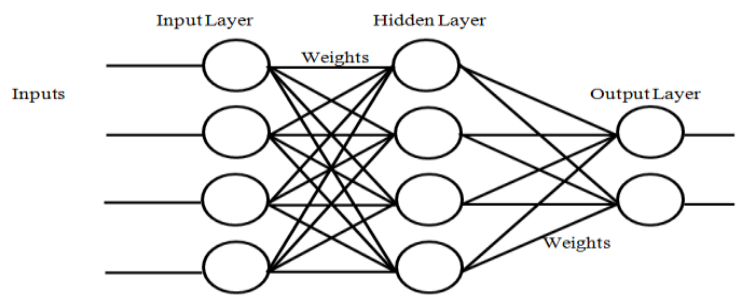

Figure 7: Block diagram of a feed-forward neural network

The hidden layer is the actual processing layer, where a transfer (or activation) functions called sigmoid calculates a layer's output from its net input. There are two sigmoid functions namely log-sigmoid and tan-sigmoid, which are used for pattern recognition. The log-sigmoid function generates outputs between 0 and 1 as the neuron's net input goes from negative to positive infinity.

The output of mathematical calculation by using sigmoid function at every neuron can be defined as follows.

$$
a_{j_{m}}=\frac{1}{1+e^{-S_{j_{m}}}}
$$

Where,

$$
S_{j_{m}}=\sum_{x=0}^{n} \quad w_{i j} a_{i_{x}}
$$

where $a_{j_{m}}$ is the activation code of receiving neuron $\mathrm{m}$ in layer $\mathrm{j}, \mathrm{S}_{\mathrm{j}}$ is the sum of the products of the activations of all relevant "emitting" nodes (i.e., the nodes in the preceding layer i) by their respective weights, and $\mathrm{w}_{\mathrm{ij}}$ is the set of all weights between layers $i$ and $j$ that are associated with vectors that feed into neuron $m$ of layer $j$.

\section{PROPERTIES OF ASSAMESE SCRIPTS}

Assamese which is the official language in Assam is a major spoken language of entire North-East India with about 30 million speakers. Assamese script is evolved from Brahmi, an ancient script of India, from which most of the Indian scripts originated through various transformations. It is written from left to right, keeping a space between two words as delimiter. Most of the character has matra in upper section of the font. There are 10 digits, 11 vowels, 41 consonants in Assamese script together with 21 three-phoneme compound characters and 143 two-phoneme compound characters. The symbol of Assamese digits and their corresponding in English are defined in table 1 .

Table 1: Assamese numerals

\begin{tabular}{|l|l|l|l|l|l|l|l|l|l|l|}
\hline Assamese & $১$ & ২ & $৩$ & 8 & $\mho$ & $৬$ & ๑ & ৮ & ১ & $\circ$ \\
\hline English & 1 & 2 & 3 & 4 & 5 & 6 & 7 & 8 & 9 & 0 \\
\hline
\end{tabular}




\section{LITERATURE REVIEW}

Richa Sharma, Arun Jain, Ritika Sharma and Jyoti Wadhwa, in the year 2013, proposed a character and digits recognition system for English language [9]. In their experiment they have used primitive morphological operations for preprocessing of image and a decision tree classifier algorithm is designed for classification of digits. According to their claim the average digit recognition accuracy is $90.1 \%$. A neural network based Gujarati handwritten numeral recognition is proposed by Apurva A. Desai [3]. At first, contrast is adjusted using adaptive histogram equalization algorithm to efficiently deal with various color ink that is used for writing. Using median filter smoothing is done on each $3 \times 3$ neighborhood pixels of the image. After preprocessing digits are converted into16x16 pixels. The X-profile, Y-profile, diagonall profile and diagonal 2 profile of each digit are used as feature to identify various digits considering $3 \times 3$ boxes. Thus various profile (a, $\mathrm{b}, \mathrm{c}, \mathrm{d})$ vector is created for all the digits as shown in figure 8 . In order to deal with skew that may present in the training set of digit, rotation upto $10^{\circ}$ is performed about center point of the digit. A feed forward back propagation neural network of three layers with 94,50 , and 10 neurons is used for classification. The input layer accepts the $1 \times 94$ profile vector. The network trained by total 610 digits and tested with 2650 digits. They have reported that using the above architecture the accuracy obtained is $81.66 \%$.

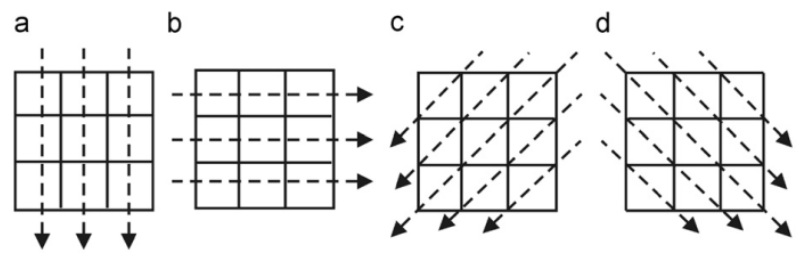

Figure 8: Various profile of $3 \times 3$ pattern matrix

Bhattacharya and Chaudhuri proposed a handwritten Bangla numeral recognition system using multi-resolution wavelet analysis with majority voting approach [11]. They extract wavelet filtering features in three different resolutions. A multi-layer perceptron (MLP) neural network is used for classification on each resolution, and then combines the classification results by majority vote. Their dataset contains 6000 samples for training, 1000 samples for validation, and another 5000 samples for testing. They reported that recognition rate is $97.16 \%$ with $0.76 \%$ rejection.

Recently, a system is designed [2] with a decision tree algorithm to recognized handwritten digits by using mathematical morphology. The dataset was taken from Kolkata ISI for testing of the proposed algorithm. The blobs and stems are used as feature for recognition of numerals. After preprocessing the image is placed at the root of the tree, where a question is asked and depending on the answer the image is placed at a particular class with blobs or without blobs, and goes to next node of the tree. Thus repeating the process image arrived at one of the ten bottom nodes and it is recognized as particular digit. Image without blobs were further process by drawing line to right, left, middle, vertically and horizontally to create blobs. The recognition rate for printed digits is claimed as $100 \%$ and for handwritten digits recognition rate is $80 \%$.

Sharma and Gupta [8] used $4 \times 4$ zone density of pixel as features and a $\mathrm{k}-\mathrm{nn}$ classifier with $\mathrm{k}=1$ for classification. They have used 10,000 and 5400 training and test patterns respectively. The best result of the experiment is claimed as $99.89 \%$ recognition rate.
Raveendra Babu and M. Radhika Mani design a system for handwritten English digits recognition by using mathematical morphology [10]. They proposed a decision tree algorithm for recognition of digits where number of blobs and stems are taken as features. According to their claim the average success rates of recognition of all digits are above $90 \%$. Their method fails to detect the broken digits with large gap.

Amitava Choudhury and Joydeep Mukherjee in the year 2013 proposed segmentation of handwritten Bangla digits [12]. At first, image is cropped and two sub images are produced; image in first line and image in the remaining lines. Then same procedure was repeated with the sub image of remaining lines until all lines were become a sub image. After that each line was segmented into numbers in between the spaces of the numerals. The proposed method will work well where numerals are unconnected; otherwise create incorrect segmentation, where there is no space between the numerals.

Our approach is different in such a way that we do not segment line and then numerals, but directly crop all the numerals using bounding box method of Matlab that does the same task.

\section{EXPERIMENTAL SETUP 6.1 Data Collection, Preprocessing and Segmentation}

In the present study, both the printed and handwritten digits are considered for recognition. The handwritten digits, printed multi font and size digits are scanned by a scanner with 300 dpi resolution. Handwritten digits are collected from 15 people written in a A4 size paper. The figure 9 shows a sample of data written by 9 people.

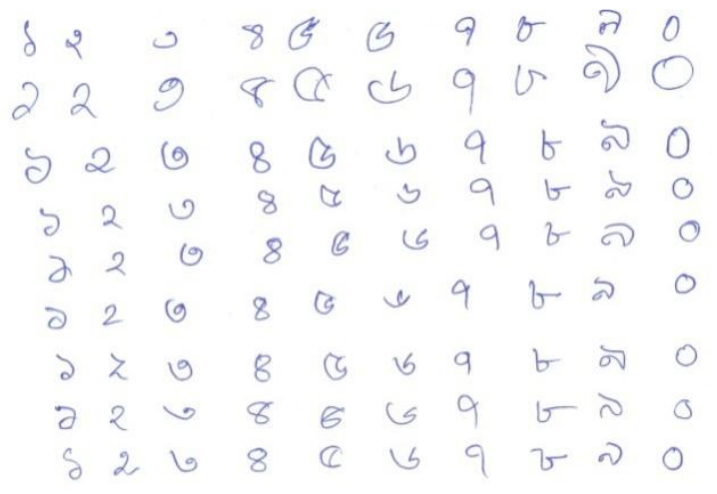

Figure 9: Sample of handwritten Assamese digits

After that image is converted to gray scale image since we do not need any color information. The gray scale image is converted to binary image with global thresholding using Otsu's method [28]. The binarization also makes the background of image uniform by removing gray values pixel which is important for further processing. In the next process, the image is morphologically opened to remove the small dots that may present in the background of image due to imperfect scanning or printing. The individual character from this clear image needs to crop before feature extraction.

After preprocessing bounding box technique is used to segment the individual image. Figure 10 depicted an image where bounding box technique is used. 


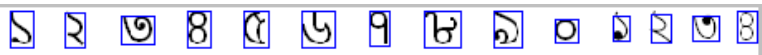

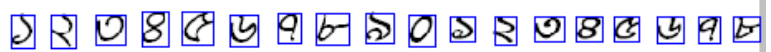

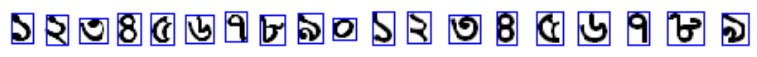

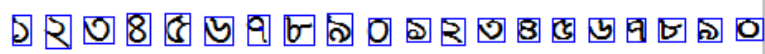

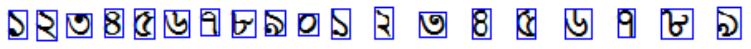

Figure 10: Applying bounding box over image

\subsection{Feature Extraction}

There are a lot of feature extraction techniques based on projection, histograms, intersections, mathematical transformation, moment, graph, geometrical shape, contour and so on. The feature extraction technique used in this experiment is zone based, which is used to obtain useful local information of patterns image like human perception. Zoning is powerful feature extraction technique used in many character recognition works. An outstanding literature about zoning method can be found in an article of D. Impedovo and G.Pirlo [6]. In this experiment static zoning method is used for recognition. In static method character image divided into many regions of $\mathrm{MxN}$, which resulting many sub image of identical shape.

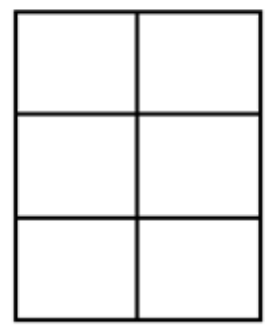

a

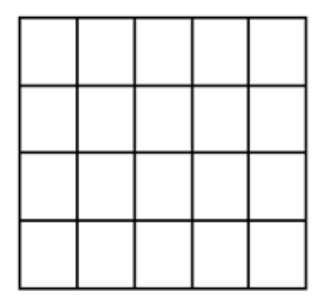

c

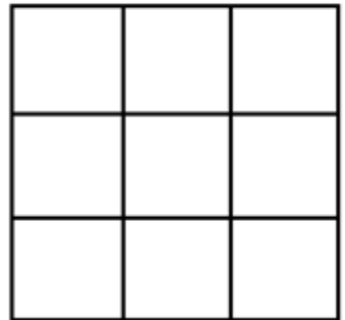

b

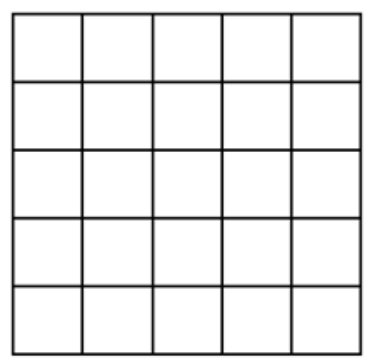

d
Figure 11: uniform zones of an image

The figure 11(a), 11(b), 11(c) and 11(d) represent $3 \times 2,3 \times 3$, $4 \times 5$ and $5 \times 5$ uniform zoning of same shape of image pattern. In this experiment uniform zoning $10 \times 10$ is used for feature extraction. The segmented image is normalized to size $70 \times 50$. So we obtained 35 element feature vector. This feature vector is provided as input to the feed forward neural network.

\section{RESULT OF EXPERIMENT}

\subsection{Network Architecture}

A four layered feed forward neural network has been used to design the network in the present study. The network consists of one input layer, two hidden layer and one output layer. The number of neurons in input layer is 35 to accept 35 element input vector. The number of neurons in $1^{\text {st }}$ and second hidden layer is 25 and 10 respectively. Number of neurons in output layer is 10 since there are 10 digits.

The parameters used in experiment one for digits are listed in Table 2. The back propagation algorithm is used for error calculation and weight adjustment of the links. Parameters used for traingdx algorithm.

Table 2: Parameter used in network

\begin{tabular}{|l|l|}
\hline Parameter & Value \\
\hline learning Rate & 0.1 \\
\hline Momentum constant & 0.95 \\
\hline Performance goal & 0.1 \\
\hline $\begin{array}{l}\text { Maximum number of } \\
\text { epochs to train }\end{array}$ & 3000 \\
\hline $\begin{array}{l}\text { Minimum Error Exist in the } \\
\text { Network }\end{array}$ & 0.001 \\
\hline $\begin{array}{l}\text { Initial weights and biased } \\
\text { term values }\end{array}$ & $\begin{array}{l}\text { Randomly Generated } \\
\text { Values Between 0 and 1 }\end{array}$ \\
\hline
\end{tabular}

The best performance of the network reached at 124 epochs with back propagation algorithm. The figure 12 depicts the best performance of the feed forward neural network.

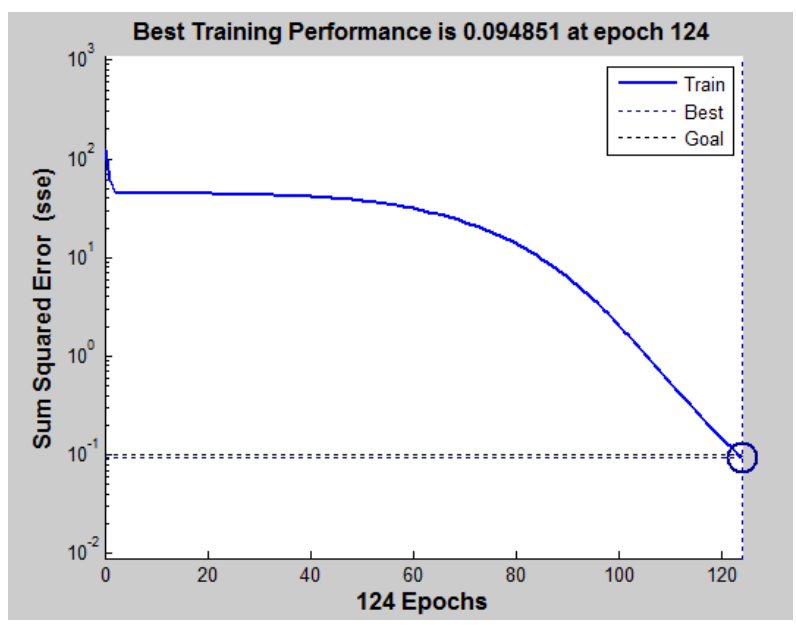

Figure 12: Best performance of feed-forward neural network

The preprocessing operations are performed before segmentation and classification of digits. Isolated digits are cropped using bounding box technique. The image are normalized into size 70x50 and uniform zone based feature is extracted from each 10x10 sub section of the cropped image, which provides 35 features. The feature vector generated in the feature extraction process is given as input to the input layer of the network and network is trained using back propagation algorithm with sum squared error 0.001 . The proposed network was trained with 500 digits, tested with 600 other new digits. The success rate for the training set is higher 
than the overall success rate. While testing the network, it is observed that the recognition rate of printed digits is $98 \%$, including multi size, bold and italics fonts. In case of handwritten digits recognition rate is $70.6 \%$.

The table 3 depicts the details of datasets used in the present study.

Table 3: Dataset used in experiment

\begin{tabular}{|c|c|c|c|}
\hline $\begin{array}{c}\text { Type of } \\
\text { Data }\end{array}$ & $\begin{array}{c}\text { Trained } \\
\text { Digits }\end{array}$ & $\begin{array}{c}\text { Tested } \\
\text { Digits }\end{array}$ & Accuracy \\
\hline Printed & 150 & 200 & $98 \%$ \\
\hline Handwritten & 350 & 400 & $70.6 \%$ \\
\hline Total & 500 & 600 & $84.3 \%$ \\
\hline
\end{tabular}

The figure 13 shows recognition rate of printed and handwritten Assamese digits.

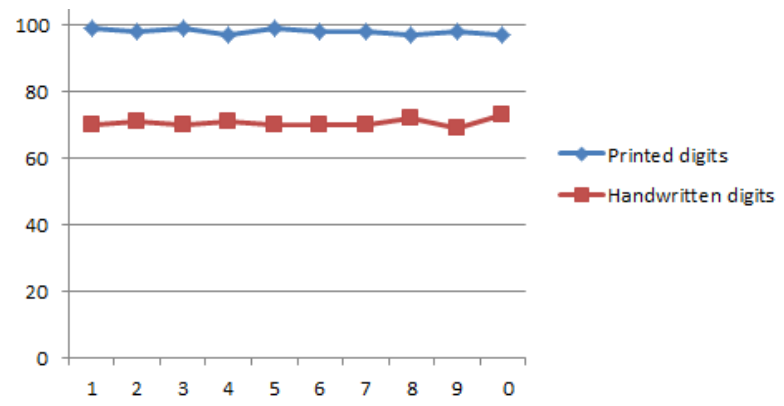

Figure 13: Recognition rate of printed and handwritten Assamese digits

\section{CONCLUSION}

In this experiment a feed forward neural network with back propagation is used for the classification of the isolated Assamese numerals. Digits are scanned from document. After preprocessing image are cropped with bounding box to obtain individual digits. Features are extracted from the each zone of the image. The recognition of this proposed system is $98 \%$ for printed digits and $70.6 \%$ for handwritten digits, which is not close to commercial application. In order to improve the performance of this system, features extraction technique has to be improved and/ or better preprocessing techniques have to be implemented.

\section{ACKNOWLEDGMENTS}

We are thankful to the department of computer science, Gauhati University for providing computing facility for this experiment.

\section{REFERENCES}

[1] Available:http://tdildc.in/index.php?option=com_vertical\&parentid=77 Accessed on 1st June 2014

[2] K. Medhi, S. K. Kalita, "Recognition of assamese handwritten numerals using mathematical morphology", Advance Computing Conference (IACC), 2014 IEEE International, India, February 2014, pp.1076-1080

[3] S. Naz, K. Hayat, M. I. Razzak, et al.: "The optical character recognition of Urdu-like cursive scripts", Pattern Recognition, 2014, vol. 47, pp. 1229-1248

[4] B. Yegnarayana, Artificial Neural Networks, Prentice Hall of India, 2004.
[5] K. L. Du, M.N.S. Swamy, "Neural Networks in a Soft computing Framework", Springer-Verlag London Limited, 2006

[6] D. Impedovo and G.Pirlo , "Zoning methods for handwritten character recognition: A survey", Pattern Recognition vol. 47, pp. 969-981, 2014

[7] J. Park, V. Govindaraju, S. N. Srihari, OCR in a hierarchical feature space, IEEE Transactions on PAMI22, vol. 4, pp. 400-407, 2000

[8] D. Sharma, D. Gupta, "Isolated handwritten digit recognition using adaptive unsupervised incremental learning technique", International Journal of Computer Applications vol. 7, pp. 27-33, 2010

[9] R. Sharma, A. Jain, R. Sharma and J. Wadhwa, "Character And Digit Recognition Aided by Mathematical Morphology", International Journal of Computer Technology \& Applications, vol. 4, pp. 828832,2013

[10] V. V. Kumar, A. Srikrishna , B. R. Babu and M. R. Mani, "Classification and recognition of handwritten digits by using mathematical morphology", Sadhana vol. 35 , pp. 419-426, 2010

[11] U. Bhattacharya, B. B. Chaudhuri, “A majority voting scheme for multi resolution recognition of hand printed numerals", in: Proceedings of the 7th International, Conference on Document Analysis and Recognition, Edinburgh, Scotland, pp. 16-20, 2003

[12] A. Choudhury and J. Mukherjee, "An Approach towards Recognition of Size and Shape Independent Bangla Handwritten Numerals", International Journal of Science and Research (IJSR), pp. 223-226, 2013

[13] R.A. Peters, T.N. Nashville, A new algorithm for image noise reduction using mathematical morphology, IEEE Transactions on Image Processing, 4 , (1995) 554-568

[14] H.K. Kwag, S.H. Kim, S.H. Jeong, G.S. Lee, Efficient skew estimation and correction algorithm for documen images, Image and Vision Computing, 20 (2002) 25-35

[15] Y. Caoa, S. Wangb, H. Lia, Skew detection and correction in document images based on straight-line fitting, Pattern Recognition Letters, 24 (2003) 18711879

[16] H. Liua, Q. Wua, H. Zhaa, X. Liuc, Skew detection for complex document images using robust borderlines in both text and non-text regions", Pattern Recognition Letters, 29 (2008) 1893-1900

[17] U. Mathur, R. Sharma, N. Srivastava, Script independent angular skew detection and correction algorithms, Signal Processing and Communication (ICSC), 2013 International Conference on, (2013) 466-469

[18] I. Marosi, Industrial OCR approaches: Architecture, algorithms, and adaptation techniques, In Proc. of SPIE, 6500 (2007) $1-10$

[19] D. Svozil, V. Kvasnicka, J. Pospichal, Introduction to multi-layer feed-forward neural networks, Chemometrics and Intelligent Laboratory Systems, 39 (1997) 43-62

[20] C.M. Bishop, Neural Networks for Pattern Recognition, Claderon Press, Oxford, 1995. 
[21] http://in.mathworks.com/help/images/noiseremoval.html? nocookie $=$ true

[22] N. Sankaran and C.V Jawahar , "Recognition of printed Devanagari text using BLSTM Neural Network", Pattern Recognition (ICPR), 21st International Conference on, Tsukuba, pp. $322-325,2012$

[23]K. S. Siddharth, M. Jangid, R. Dhir, R. Rani, "Handwritten Gurmukhi Character Recognition Using Statistical and Background Directional Distribution Features", International Journal on Computer Science and Engineering, Vol. 3, pp.2332-2345, 2011

[24]H.R Mamatha, S. Sucharitha and K. Srikanta Murthy, "Multi-font and Multi-size Kannada Character Recognition based on the Curvelets and Standard Deviation", International Journal of Computer Applications, Vol. 35, Dec. 2011

[25] A. Sampath, C. Tripti, V. Govindaru, "Freeman code based online handwritten character recognition for Malayalam using Back propagation neural networks", Advance computing: An international journal, Vol. 3, pp. 51-58, Jul. 2012

[26]A. Kokku and S. Chakravarthy, "A Complete OCR System for Tamil Magazine Documents", Guide to OCR for Indic Scripts, Springer London, pp. 147-162, 2010

[27] S. Dewan, S. Chakravarthy, "A System for Offline Character Recognition Using Auto-encoder Networks", 19th International Conference, ICONIP 2012, Doha, Qatar, Nov. 2012, Vol. 7666, pp. 91-99, 2012

[28] N. Otsu, A threshold selection method from gray level histograms. IEEE Transactions on Systems, Man and Cybernetics, 9(1), pp. 62-66,1979
[29] S. Bag, G. Harit, P. Bhowmick, "Recognition of Bangla compound characters using structural decomposition", Pattern Recognition, Vol. 47, pp.1187-1201, 2014

[30] S. Barman, D. Bhattacharyya, S. Jeon, T. Kim, H. Kim, "A New Experiment on Bengali Character Recognition", International Conference, UCMA 2010, Miyazaki, Japan, Jun. 2010. Proceedings, pp. 20-28

[31] A. Choudhury and J. Mukherjee, "An Approach towards Recognition of Size and Shape Independent Bangla Handwritten Numerals", International Journal of Science and Research (IJSR), Vol. 2, pp. 223-226, Jan. 2013

[32] N. Das, K. Acharya, R. Sarkar, S. Basu, M. Kundu, M. Nasipuri, "A Novel GA-SVM Based Multistage Approach for Recognition of Handwritten Bangla Compound Characters", Advances in Intelligent and Soft Computing, Vol. 132, pp. 145-152, 2012

[33] M. Hangarge, G. Mukarambi and B. V. Dhandra, "South Indian Handwritten Script Identification at Block Level from Trilingual Script Document Based on Gabor Features", Proceedings of the First International Conference, ICMCCA, Dec. 13-15, 2012, Vol. 213, pp. 25-33

[34] S. Dewan, S. Chakravarthy, "A System for Offline Character Recognition Using Auto-encoder Networks", 19th International Conference, ICONIP 2012, Doha, Qatar, Nov. 2012, Vol. 7666, pp. 91-99, 2012

[35] V. Rasagna, K. J. Jinesh and C. V. Jawahar, "On Multifont Character Classification in Telugu", International Conference, ICISIL 2011, Patiala, India, Mar., 2011, Vol. 139, pp. 86-91 